\title{
Plasma homocysteine level is a risk factor for osteoporotic fractures in elderly patients
}

\author{
This article was published in the following Dove Press journal: \\ Clinical Interventions in Aging \\ 18 August 2016 \\ Number of times this article has been viewed
}

\author{
Yuefeng Zhu' \\ Jie Shen ${ }^{2}$ \\ Qun Cheng ${ }^{3}$ \\ Yongqian Fan' \\ Weilong Lin' \\ 'Department of Orthopedics, \\ ${ }^{2}$ Department of Pharmacy, \\ ${ }^{3}$ Department of Osteoporosis, \\ Huadong Hospital, Fudan University, \\ Shanghai, People's Republic of China
}

Correspondence: Yuefeng Zhu Department of Orthopedics, Huadong Hospital, Fudan University, No 21I,

West Yanan Road, Shanghai 200040, People's Republic of China

Tel +86 2I 62483 I80 ext 7I50I

Email zhuyuefenghd@vip.163.com
Objective: To study the relationship of plasma homocysteine (Hcy), bone turnover biomarkers (BTB), and bone mineral density (BMD) with osteoporotic fracture (OPF) in elderly people.

Methods: Eighty-two patients (aged 65 years or older) admitted to our orthopedics department between October 2014 and May 2015 were randomly divided into three groups: 1) OPF group: 39 cases with the mean age $81.82 \pm 5.49$ years, which included 24 females and 15 males; 2 ) highenergy fracture (HEF) group: 22 cases with the mean age $78.88 \pm 5.75$ years, which included 16 females and six males; 3 ) non-bone-fracture group: 21 cases with mean age $79.75 \pm 5.47$ years without bone fracture, which included 14 females and seven males. Plasma Hcy, BTB, and BMD were measured. Analysis of variance and multiple regression analysis were used in the statistical analysis.

Results: There was no significant difference in either age or sex among the three groups. There were significant differences in plasma Hcy and hip BMD between the OPF and HEF groups; there was also significant difference in plasma Hcy, 25-(OH) Vit D, and hip BMD between the OPF and non-fracture groups. There was no difference in lumbar spine BMD between the OPF group and the other two groups. There was no significant difference in plasma Hcy, 25-(OH) Vit D, hip or lumbar spine BMD between the HEF and non-fracture group. There was no significant difference in procollagen type I N-propeptide of type I collagen, serum C-terminal cross-linking telopeptide of type I collagen, and parathyroid hormone among the three groups. Plasma Hcy was linearly correlated with age and serum C-terminal cross-linking telopeptide of type I collagen, but not correlated with either hip or lumbar spine BMD or any other BTBs.

Conclusion: In this study, we found that the plasma Hcy level in elderly patients with OPF is higher than that of nonosteoporotic patients. It is not correlated with BMD, but positively correlated with bone resorption markers. An increased Hcy level appears to be a risk factor for OPFs in elderly people.

Keywords: elderly patient, osteoporosis, fracture, homocysteine, bone mineral density, bone turnover marker

\section{Introduction}

With the increase of human life expectancy and the arrival of an aging society, osteoporosis has become an important human health problem. Osteoporosis is a wholebody bone disease characterized by decreased bone strength and increase in the risk of fracture caused by low bone mass and bone microstructural damage. ${ }^{1}$ A serious consequence of osteoporosis is the occurrence of osteoporotic fracture (OPF), that is, a fracture induced by minor trauma or a daily routine activity. ${ }^{1}$ OPF is a serious threat to the health of elderly population, as it can reduce their quality of life and significantly increase the morbidity and mortality. ${ }^{2}$ For example, the mortality will increase to $12 \%-35 \% 1$ year after hip fracture. ${ }^{3}$ 
Homocysteine (Hcy) is a nonprotein amino acid that is synthesized from methionine and either recycled back into methionine or converted into cysteine with the aid of the B-group vitamins. Increase in plasma Hcy has been linked to the increased incidence of osteoporosis-related fractures. ${ }^{46}$ However, the exact mechanism of how Hcy causes OPF is still unclear. Previous literature have reported contradictory findings about the relationship between Hcy and bone mineral density (BMD), ${ }^{6-11}$ some reported reverse, mixed, or no relationships at all. A meta-analysis study in a female population showed no significant association between Hcy and BMD. ${ }^{12}$ However, a most recent cross-sectional study performed in postmenopausal females showed that serum Hcy levels were significantly higher in osteoporotic females compared to the other BMD groups, and were inversely related to lumbar spine and femoral neck BMD ${ }^{13}$ In the past, it was hypothesized that Hcy was related to bone collagen cross-linking and might weaken the bone structure. ${ }^{14}$ Based on the above findings, recently, there was a study to see whether lowering Hcy with combined vitamin B-12 and folic acid supplementation will reduce fracture risk in the elderly population with elevated Hcy level. It was found that combined vitamin B-12 and folic acid supplementation had no effect on OPF incidence in the elderly population. ${ }^{15}$

There are few studies about the relationship between Hcy and bone metabolism, and the relationship between Hcy and BMD reported in the literature is confusing. Therefore, the aim of this study is to explore the relationship between senile OPFs and plasma concentrations of Hcy.

\section{Materials and methods Study subjects}

Eight-two patients aged $>65$ years who were admitted to the orthopedic department at Huadong Hospital in Shanghai, People's Republic of China, between October 2014 and May 2015 were enrolled in this study, and their ages were $80.61 \pm 5.61$ years. The patients were divided into three groups: 1) OPF group: 39 cases with the mean age $81.82 \pm 5.49$ years, 24 females and 15 males, which included 26 cases of hip fractures, eleven cases of thoracolumbar fractures, and two cases of distal radius fractures; 2) high-energy fracture (HEF) group: 22 cases with the mean age $78.88 \pm 5.75$ years, which included 16 females and six males; 3 ) non-bone-fracture (NF) group: 21 cases with the mean age $79.75 \pm 5.47$ years without bone fracture, 14 females and seven males, which included 15 cases of degenerative lumbar spinal stenosis, three cases of cervical spine disease and three cases of knee osteoarthritis.

The inclusion criteria were the following: 1) Diagnosis with either OPF HEF, or without fracture but with bone disease that needs inpatient treatment. 2) Age is over 65 years. 3) Voluntary participation. The exclusion criteria were the following: 1) Lower extremity arterial disease confirmed by lower extremity peripheral artery intravascular ultrasound or magnetic resonance imaging (MRI). 2) Severe cardiovascular disease and heart failure based on history and cardiac enzyme tests. 3) Cognitive impairment based on mini-mental state examination (MMSE) scale test. ${ }^{16}$

This study was approved by the Huadong Hospital ethics committee, and all study subjects signed an informed consent form to voluntarily participate in this study.

\section{Methods}

Blood was drawn from all patients after overnight fasting and placed in anticoagulant tubes containing ethylenediaminetetraacetic acid. The blood samples were incubated at $37^{\circ} \mathrm{C}$ water bath for half an hour, centrifuged at $4^{\circ} \mathrm{C}$, and the supernatant was collected. Plasma Hcy concentration was measured using a automatic biochemical analyzer (Olympus AU640, Olympus Corporation, Tokyo, Japan) and Hcy assay kit (normal reference values $\leq 15 \mu \mathrm{mol} / \mathrm{L}$ ).

Plasma biochemical markers of bone turnover recommended by the International Osteoporosis Foundation were measured simultaneously, including markers of bone formation such as procollagen type I intact $\mathrm{N}$-terminal propeptide, and bone resorption markers such as serum $\mathrm{C}$-terminal crosslinking telopeptide of type I collagen (S-CTX), ${ }^{1}$ as well as plasma levels of 25-OH Vit D.

BMD was measured by dual-energy X-ray assessment absorptiometry. Two body parts - the femoral neck and lumbar spine (L1-L4) - were used for BMD measurement.

\section{Statistical analysis}

Statistical analysis was performed using SPSS22.0 software (SPSS Inc., Chicago, IL, USA). Numerical data were expressed as mean \pm standard deviation and analysis of variance was used to compare the differences among the three groups; categorical data were expressed as percentage and Chi-square test was used to compare the differences between the two groups; multiple regression analysis was used to study the relationship between plasma Hcy level and bone metabolism, and hip and lumbar spine BMD. $P<0.05$ was regarded as statistically significant.

\section{Results}

As shown in Table 1, there was no statistical difference in age and sex among the three groups. However, there was significant difference in plasma Hcy level among the three groups $(P=0.000)$. In terms of bone metabolism, there was also 
Table I General characteristics, plasma Hcy level, BTB, and BMD in the three groups

\begin{tabular}{llllll}
\hline Factors & OPF group & HEF group & NF group & F-value & P-value \\
\hline Age $($ years old) & $81.82 \pm 5.49$ & $78.88 \pm 5.75$ & $79.75 \pm 5.47$ & 2.179 & 0.122 \\
Sex (female \%) & 61.54 & 72.73 & 66.67 & - & 0.385 \\
Hcy $(\mu \mathrm{mol} / \mathrm{L})$ & $17.21 \pm 5.99 *$ & $10.82 \pm 3.60$ & $12.91 \pm 4.32$ & 9.483 & 0.000 \\
S-CTX $(\mathrm{pg} / \mathrm{mL})$ & $621.89 \pm 383.19$ & $474.81 \pm 220.71$ & $473.57 \pm 278.22$ & 1.427 & 0.248 \\
Osteocalcin $(\mathrm{ng} / \mathrm{mL})$ & $16.57 \pm 11.08$ & $13.87 \pm 5.28$ & $16.43 \pm 8.61$ & 0.540 & 0.586 \\
PINP $(\mathrm{ng} / \mathrm{mL})$ & $72.40 \pm 60.18$ & $74.37 \pm 55.96$ & $53.14 \pm 26.23$ & 0.676 & 0.513 \\
PTH $(\mathrm{pg} / \mathrm{mL})$ & $69.75 \pm 143.88$ & $43.66 \pm 29.24$ & $44.11 \pm 20.38$ & 0.437 & 0.648 \\
25-(OH) Vit D $(\mathrm{ng} / \mathrm{mL})$ & $13.77 \pm 7.85^{\#}$ & $18.79 \pm 4.84$ & $18.70 \pm 3.19$ & 4.792 & 0.012 \\
LI-L4 BMD $\left(\mathrm{g} / \mathrm{cm}^{2}\right)$ & $0.79 \pm 0.17^{\#}$ & $0.99 \pm 0.21$ & $0.95 \pm 0.18$ & 3.750 & 0.037 \\
Hip BMD $\left(\mathrm{g} / \mathrm{cm}^{2}\right)$ & $0.65 \pm 0.11^{*}$ & $0.81 \pm 0.13$ & $0.85 \pm 0.24$ & 5.473 & 0.009 \\
\hline
\end{tabular}

Notes: Data presented as mean \pm standard deviation unless stated otherwise. ${ }^{* P}<0.01$ compared with either HEF or NF group, ${ }^{\sharp P}<0.05$ compared with either HEF or NF group. "-" indicates no data.

Abbreviations: BMD, bone mineral density; BTB, bone turnover biomarker; Hcy, homocysteine; HEF, high-energy fracture; LI-L4, lumbar spine; NF, non-fracture; OPF, osteoporotic fracture; PINP, procollagen type I intact N-terminal propeptide; PTH, parathyroid hormone; S-CTX, serum C-terminal cross-linking telopeptide of type I collagen.

significant difference in plasma 25-(OH) Vit D level among the three groups $(P=0.012)$; however, there was no statistically significant difference in plasma levels of procollagen type I intact N-terminal propeptide, S-CTX, osteocalcin, and parathyroid hormone among the three groups. In terms of bone density, there was significant difference in hip and lumbar spine BMD among the three groups $(P=0.009$ and $P=0.0037$, respectively).

As shown in Table 2, plasma level of Hcy in OPF group was significantly higher than those in both HEF and NF groups ( $P<0.05$ for both comparisons), but plasma levels of 25-(OH) Vit D and hip BMD in OPF group were significantly lower than those in both HEF and NF groups ( $P<0.05$ for all comparisons). There was no significant difference in lumbar BMD among the three groups. There was no significant difference in plasma Hcy, 25-(OH) Vit D, hip BMD and lumbar BMD $(P=0.498, P=0.999$, $P=0.869$, and $P=0.935$, respectively) between the HEF and NF groups.

As shown in Table 3, plasma Hcy level was positively and linearly correlated with age $(r=0.386, P<0.01)$, but not correlated with either hip BMD $(r=-0.221, P=0.183)$ or lumbar spine BMD ( $r=-0.106, P=0.533)$. In the meantime, plasma Hcy level was also positively and linearly correlated with S-CTX ( $r=0.324, P=0.003)$, but not to any other bone metabolism marker.

Table 2 Comparisons of plasma Hcy, 25-(OH) Vit D, hip and lumbar spine BMD among the three groups

\begin{tabular}{lllll}
\hline Comparisons & Hcy & 25(OH) D & $\begin{array}{l}\text { Hip } \\
\text { BMD }\end{array}$ & $\begin{array}{l}\text { Lumbar } \\
\text { spine BMD }\end{array}$ \\
\hline OPF vs HEF group & 0.000 & 0.033 & 0.034 & 0.052 \\
OPF vs NF group & 0.024 & 0.048 & 0.022 & 0.143 \\
HEF vs NF group & 0.498 & 0.999 & 0.869 & 0.935 \\
\hline
\end{tabular}

Abbreviations: BMD, bone mineral density; Hcy, homocysteine; HEF, high-energy fracture; NF, non-fracture; OPF, osteoporotic fracture.

\section{Discussion}

Osteoporosis and fall are the two major risk factors for OPF. Bone strength is determined by BMD and bone quality. BMD represents approximately $70 \%$ of bone strength and is a commonly used quantitative indicator in clinical diagnosis of osteoporosis and prediction of the risk of OPF. ${ }^{1}$ Studies have shown that one standard deviation decrease in BMD was accompanied with 2- to 2.5 -fold increase in fracture risk. ${ }^{14}$ This study also showed that, compared with the other two groups, hip BMD in the osteoporotic group is significantly lower, which also indicates that bone density can be used as a predictive indicator for OPF. There was no significant difference in lumbar spine BMD among the three groups, which is probably due to the finding that degenerative lumbar hyperplasia often interferes with normal BMD in lumbar spine. BMD only reflects the level of bone mineralization. We found in this study that the plasma Hcy level in the OPF group was significantly higher than those in the other two groups. But in literature, the relationship between plasma Hcy and BMD remains unclear, and conflicting results have been reported in the past. ${ }^{6-11}$ We did not find any correlation

Table 3 Multiple regression analysis of plasma Hcy level with all other parameters

\begin{tabular}{lll}
\hline Factor & Hcy & P-value \\
\cline { 2 - 2 } & $\boldsymbol{R}$ & \\
\hline Age (years old)* & 0.386 & $<0.01$ \\
S-CTX (pg/mL)* & 0.324 & 0.003 \\
Osteocalcin $(\mathrm{ng} / \mathrm{mL})$ & 0.115 & 0.307 \\
PINP $(\mathrm{ng} / \mathrm{mL})$ & 0.039 & 0.732 \\
25(OH) D $(\mathrm{ng} / \mathrm{mL})$ & -0.211 & 0.058 \\
LI-L4 BMD $\left(\mathrm{g} / \mathrm{cm}^{2}\right)$ & -0.106 & 0.533 \\
Hip BMD $\left(\mathrm{g} / \mathrm{cm}^{2}\right)$ & -0.221 & 0.183 \\
\hline
\end{tabular}

Note: $* p<0.01$.

Abbreviations: 25(OH) D, 25-hydroxy vitamin D; BMD, bone mineral density; Hcy, homocysteine; PINP, procollagen type I intact N-terminal propeptide; S-CTX, serum C-terminal cross-linking telopeptide of type I collagen. 
between plasma Hcy levels and BMD in this study either. Therefore, we assume that the impact of plasma Hcy level on bone fracture is through its effect on bone quality. It has been reported in previous studies that plasma Hcy can enhance osteoclast activity, inhibit osteoblast activity, and activate matrix metalloproteinases activity by increasing the production of reactive oxygen species. It can therefore interfere with collagen cross-linking, cause degradation of extracellular bone matrix, and reduce the strength of bone microarchitecture structure, which will result in damage of biomechanical properties of the bone, reduced bone quality, and increased risk of fractures. ${ }^{14} \mathrm{We}$ also found that plasma Hcy and S-CTX has a weak correlation. Serum S-CTX is a relatively sensitive marker for bone resorption, which is recommended by the International Osteoporosis Foundation; the correlation of Hcy with S-CTX reported in our study also supports the previous finding that Hcy could increase osteoblast activity. Some researchers believe that Hcy can change the properties of bone through mitochondria-mediated pathway. It has been confirmed that there were mitochondrial abnormalities in hyperhomocysteinemia, and mitochondria play a very important role in the processing of reactive oxygen species.

Fall is another important risk factor for OPFs. ${ }^{1,2,17,18}$ Sideways fall can increase the risk of hip fracture three- to fivefold, and if the fall affects the proximal greater trochanter of the femur, then the risk of hip fracture will increase by approximately 30 -fold. ${ }^{17}$ Some researchers have analyzed the incidence rate of low-energy OPFs in a population aged $>50$ years and found that the femoral neck fracture rate was $95 \%$, the rates of fracture in other locations were $62 \%-94 \% .{ }^{19}$ Fall can be caused by many reasons, including environmental factors, health factors, neuromuscular factors, and so on. ${ }^{1}$ Health factors include insufficient vitamin $\mathrm{D}$ (serum $25-(\mathrm{OH})$ Vit $\mathrm{D}<30 \mathrm{ng} / \mathrm{mL}) .{ }^{1} \mathrm{We}$ found in this study that, in the OPF group, $25(\mathrm{OH})$ Vit D level was significantly lower than those of the control groups. In recent years, studies have shown that Hcy was associated with weakening of muscle functions. ${ }^{20,21}$ Cysteine is the mediator of a number of key metabolic processes, such as demethylation and antioxidation function in the cell. Metabolic imbalance of cysteine will affect gene expression and cell signaling (including G protein-coupled receptor signaling), and damage the sympathetic and parasympathetic function of blood vessels in skeletal muscles as well as muscle function. It was previously reported that elevated Hcy was negatively correlated with the physical capacity in the elderly. ${ }^{22,23}$ The enhanced muscle-specific creatine phosphokinase activity further proved that Hcy could damage skeletal muscle. ${ }^{24}$ Other studies suggested that Hcy could damage muscle function and integrity, leading to senile weakness. ${ }^{25}$ Cysteine was observed to stimulate the production of cellular superoxide radicals and induction of superoxide dismutase in endothelial cells. ${ }^{26}$ Hypomethylation can also affect the remodeling and repair of skeletal muscle. ${ }^{27,28}$ Hcy could potentially limit the supply of creatinine in liver, reduce muscle endurance, and increase muscle damage. ${ }^{29-32}$ Excess Hcy may affect nitric oxide signaling and limit the hemodynamics in muscles and blood vessels, resulting in fatigue, ischemia, and decreased body endurance. ${ }^{33}$ It was found that the elderly with high plasma Hcy levels had poor exercise tolerance. Recently, there was a report that Hcy could reduce connexin expression in the blood vessels of skeletal muscles and interfere with vasodilation and tissue perfusion. ${ }^{34}$ Hcy was also shown to be able to cause abnormal endoplasmic reticulum stress, resulting in muscle disease. ${ }^{35,36}$ Precise evaluation of the role of Hcy in muscle diseases will provide new ways to treat falls caused by bone or skeletal muscle weakness in elderly patients.

\section{Conclusion}

In summary, with the accelerated aging of the population, the incidence of osteoporosis and OPF has been rising; consequently, finding ways of reducing and delaying OPFs is an urgent task. We found in this study that plasma Hcy level in patients with OPFs was significantly higher than that in patients with HEF or no fracture. Plasma Hcy level is not correlated with BMD in patients, but is correlated with osteoclast activity markers in bone metabolism. Plasma Hcy level can be used as an important risk factor for OPF in the elderly population. Reducing plasma Hcy level can improve bone quality and reduce the risk of falling in elderly patients secondary to bone or skeletal muscle weakness, thereby reducing the risk of OPFs in the elderly. How to predict the occurrence of OPFs is the direction for future research.

\section{Disclosure}

The authors report no conflicts of interest in this work.

\section{References}

1. Osteoporosis treatment guidelines 2011 edition by Osteoporosis and Bone Mineral Disease branch, Chinese Medical Association. Chin J Osteo Bone Min Dis. 2011;4(1):2-17. Chinese.

2. Huang G. Clinical characteristics of osteoporotic fracture. Chin J Orthopedics. 2008;28(1):74-77.

3. Roth T, Kammerlander C, Gosch M, Luger TJ, Blauth M. Outcome in geriatric fracture patients and how it can be improved. Osteoporosis Int. 2010;21(Suppl 4):S615-S619.

4. Leboff MS, Narweker R, LaCroix A, et al. Homocysteine levels and risk of hip fracture in postmenopausal women. J Clini Endocrinol Metab. 2009;94(4):1207-1213. 
5. McLean RR, Jacques PF, Selhub J, et al. Homocysteine as a predictive factor for hip fracture in older persons. $N$ Engl J Med. 2004;350(20): 2042-2049.

6. van Meurs JB, Dhonukshe-Rutten RA, Pluijm SM, et al. Homocysteine levels and the risk of osteoporotic fracture. N Engl J Med. 2004;350(20): 2033-2041.

7. Gjesdal CG, Vollset SE, Ueland PM, et al. Plasma total homocysteine level and bone mineral density: the Hordaland homocysteine Study. Arch Intern Med. 2006;166(1):88-94.

8. Gerdhem P, Ivaska KK, Isaksson A, et al. Associations between homocysteine, bone turnover, BMD, mortality, and fracture risk in elderly women. J Bone Miner Res. 2007;22(1):127-134.

9. Bucciarelli P, Martini G, Martinelli I, et al. The relationship between plasma homocysteine levels and bone mineral density in postmenopausal women. Eur J Intern Med. 2010;21(4):301-305.

10. Cagnacci A, Baldassari F, Rivolta G, Arangino S, Volpe A. Relation of homocysteine, folate, and vitamin B12 to bone mineral density of postmenopausal women. Bone. 2003;33(6):956-959.

11. Perier MA, Gineyts E, Munoz F, Sornay-Rendu E, Delmas PD. Homocysteine and fracture risk in postmenopausal women: the OFELY study. Osteoporosis Int. 2007;18(10):1329-1336.

12. van Wijngaarden JP, Doets EL, Szczecinska A, et al. Vitamin B12, folate, homocysteine, and bone health in adults and elderly people: a systematic review with meta-analyses. J Nutr Metab. 2013;2013:486186.

13. Bahtiri E, Islami H, Rexhepi S, et al. Relationship of homocysteine levels with lumbar spine and femur neck BMD in postmenopausal women. Acta Reumatol Port. 2015;40(4):355-362.

14. Lubec B, Fang-Kircher S, Lubec T, Blom HJ, Boers GH. Evidence for McKusick's hypothesis of deficient collagen cross-linking in patients with homocystinuria. Biochim Biophys Acta. 1996;1315(3):159-162.

15. van Wijngaarden JP, Swart KM, Enneman AW, et al. Effect of daily vitamin B-12 and folic acid supplementation on fracture incidence in elderly individuals with an elevated plasma homocysteine concentration: B-PROOF, a randomized controlled trial. Am J Clin Nutr. 2014;100(6): 1578-1586.

16. Folstein MF, Folstein SE, McHugh PR. "Mini-mental state". A practical method for grading the cognitive state of patients for the clinician. J Psychiatr Res. 1975;12(3):189-198.

17. Jarvinen TL, Sievanen H, Khan KM, Heinonen A, Kannus P. Shifting the focus in fracture prevention from osteoporosis to falls. BMJ. 2008;336(7636):124-126.

18. Dargent-Molina P, Favier F, Grandjean H, Baudoin C, Schott AM, Hausherr E, et al. Fall-related factors and risk of hip fracture: the EPIDOS prospective study. Lancet. 1996;348(9021):145-149.

19. Morrison A, Fan T, Sen SS, Weisenfluh L. Epidemiology of falls and osteoporotic fractures: a systematic review. Clinicoecon Outcomes Res. 2013;5:9-18

20. Kolling J, Scherer EB, Siebert C, et al. Homocysteine induces energy imbalance in rat skeletal muscle: is creatine a protector? Cell Biochem Funct. 2013;31(7):575-584.

21. McDermott MM, Ferrucci L, Guralnik JM, et al. Elevated levels of inflammation, d-dimer, and homocysteine are associated with adverse calf muscle characteristics and reduced calf strength in peripheral arterial disease. J Am Coll Cardiol. 2007;50(9):897-905.
22. Kado DM, Bucur A, Selhub J, Rowe JW, Seeman T. Homocysteine levels and decline in physical function: MacArthur studies of successful aging. Am J Med. 2002;113(7):537-542.

23. van Schoor NM, Swart KM, Pluijm SM, et al. Cross-sectional and longitudinal association between homocysteine, vitamin B12 and physical performance in older persons. Eur J Clin Nutr. 2012;66(2):174-181.

24. Miller A, Mujumdar V, Shek E, et al. Hyperhomocyst(e)inemia induces multiorgan damage. Heart Vessels. 2000;15(3):135-143.

25. Biezanowska-Kopec R, Leszczynska T. The effect of methionine supplementation of the AIN-93G semi-synthetic diet on the levels of homocysteine and lipids in experimental rats. J Nutr. 2012;16(4):395-400.

26. Lang D, Kredan MB, Moat SJ, Hussain SA, Powell CA, Bellamy MF, et al. Homocysteine-induced inhibition of endothelium-dependent relaxation in rabbit aorta: role for superoxide anions. Arterioscler Throm Vasc Biol. 2000;20(2):422-427.

27. Acharyya S, Sharma SM, Cheng AS, et al. TNF inhibits Notch-1 in skeletal muscle cells by Ezh2 and DNA methylation mediated repression: implications in duchenne muscular dystrophy. PloS One. 2010; 5(8): 12479.

28. Caudill MA, Wang JC, Melnyk S, et al. Intracellular S-adenosylhomocysteine concentrations predict global DNA hypomethylation in tissues of methyl-deficient cystathionine beta-synthase heterozygous mice. J Nutr. 2001;131(11):2811-2818.

29. Wallimann T, Tokarska-Schlattner M, Schlattner U. The creatine kinase system and pleiotropic effects of creatine. Amino Acids. 2011;40(5):1271-1296.

30. Stead LM, Au KP, Jacobs RL, Brosnan ME, Brosnan JT. Methylation demand and homocysteine metabolism: effects of dietary provision of creatine and guanidinoacetate. Am J Physiol Endocrinol Metab. 2001;281(5):E1095-E1100.

31. Brock TA, Alexander RW, Ekstein LS, Atkinson WJ, Gimbrone MA. Angiotensin increases cytosolic free calcium in cultured vascular smooth muscle cells. Hypertension. 1985;7(3 Pt 2):I105-I109.

32. Maron BA, Loscalzo J. The treatment of hyperhomocysteinemia. Annu Rev Med. 2009;60:39-54.

33. Dankner R, Chetrit A, Dror GK, Sela BA. Physical activity is inversely associated with total homocysteine levels, independent of C677T MTHFR genotype and plasma B vitamins. Age (Dordr). 2007;29(4): 219-227.

34. Givvimani S, Narayanan N, Armaghan F, Pushpakumar S, Tyagi SC. Attenuation of conducted vasodilation in skeletal muscle arterioles during hyperhomocysteinemia. Pharmacology. 2013;91(5-6):287-296.

35. Deldicque L, Hespel P, Francaux M. Endoplasmic reticulum stress in skeletal muscle: origin and metabolic consequences. Exerc Sport Sci Rev. 2012;40(1):43-49.

36. Rayavarapu S, Coley W, Nagaraju K. Endoplasmic reticulum stress in skeletal muscle homeostasis and disease. Curr Rheumatol Rep. 2012; 14(3):238-243.
Clinical Interventions in Aging

\section{Publish your work in this journal}

Clinical Interventions in Aging is an international, peer-reviewed journal focusing on evidence-based reports on the value or lack thereof of treatments intended to prevent or delay the onset of maladaptive correlates of aging in human beings. This journal is indexed on PubMed Central, MedLine,

\section{Dovepress}

CAS, Scopus and the Elsevier Bibliographic databases. The manuscript management system is completely online and includes a very quick and fair peer-review system, which is all easy to use. Visit http://www.dovepress. com/testimonials.php to read real quotes from published authors. 